\title{
Molecular evolutionary lines in the formation of indole alkaloids derived from secologanin
}

\author{
László F. Szabó \\ Department of Organic Chemistry, Semmelweis University, Högyes utca 7, H-1092 Budapest, \\ Hungary \\ E-mail: Szalasz@szerves.sote.hu
}

Dedicated to Prof. Csaba Szántay on the occasion of his $80^{\text {th }}$ birthday

\begin{abstract}
Generation of the strictosidine $\rightarrow$ stemmadenine $\rightarrow$ ellipticine/olivacine and the strictosidine $\rightarrow$ vincadifformine $\rightarrow$ rhazinilam/rhazinal molecular evolutionary lines indicated the multistep bioorganic interactions in the frame of indole alkaloids derived from secologanin. Narrow structural elements, detailed chemotaxonomic data and standard organic reaction mechanisms, together with computer search of the DNP databases contributed considerably to the knowledge of the chemical background of this important class of natural products and detected several longrange electronic connections between the nitrogen atoms in these molecules.
\end{abstract}

Keywords: Indole alkaloids, corynanthean, malindan, computer-searched, database, chemotaxonomic

\section{Introduction}

The molecular history of natural products is written in their structure. The study of their biogenesis should detect it. In the last century, especially in the field of the indole alkaloids, large efforts were made to get more detailed information about their formation. ${ }^{1-4}$ The main elements of this work are isolation and structure determination of the natural products, use of isotope-labelled compounds and isolated enzymes, and recently genomic methods as well. Elaboration of synthetic and biomimetic chemical reactions provided also useful support to this work. However, many of these methods are slow, time, material and energy consuming. Often it is difficult or even impossible to prove if reactions discovered or applied under abiotic conditions do really run in cells or organisms. Recently, the construction of large chemical data bases offer an alternative approach. The living cell may be considered as a chemical reactor in which the natural products are formed in parallel and/or consecutive steps from the educt- 
precursors. Isolated compounds from the same cells, species, genera and/or families may be guide-posts in this process. And in the case when highly reactive or sensitive intermediates could not be isolated in intact form, they can be postulated according to the standard organic reaction mechanisms.

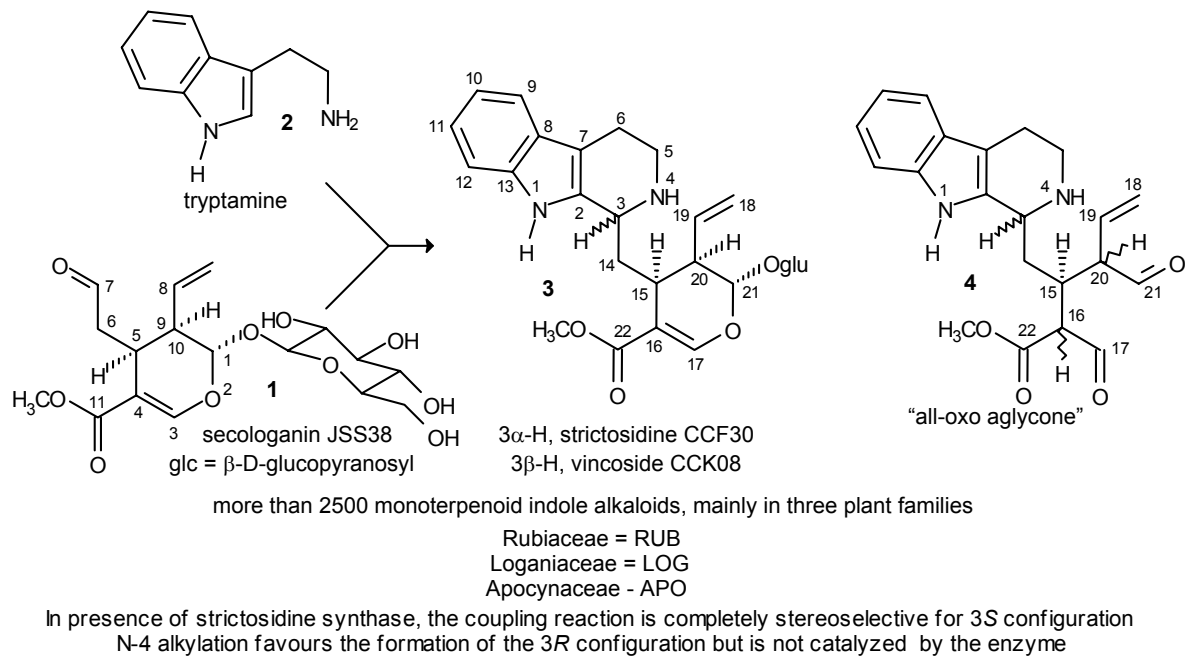

Scheme 1. The coupling reaction.

The group of indole alkaloids derived from secologanin seems to be a proper subject for such an approach. ${ }^{1-4}$ More than 2500 isolated representatives of them are formed mainly in three plant families Apocynaceae (APO), Loganiaceae (LOG) and Rubiaceae (RUB) from two building blocks secologanin $\mathbf{1}$ and tryptamine $\mathbf{2}$ through a single precursor strictosidine $\mathbf{3}$ (in this paper they are considered as indole alkaloids; however, sesqui- and dimer derivatives are temporarily disregarded). The narrow relatedness of the structures, the presence or absence of common structural elements, ring systems, functional groups, sesquimer and dimer alkaloids and stable centers of chirality ${ }^{5,6}$ give important contributions to this work. And in addition, the chemotaxonomical data provide a strong support as well. It was supposed that these circumstances give a strong internal consistency to the whole group of indole alkaloids, where the results obtained in the different subgroups should mutually strengthen each others.

The aim of the present paper is to show that rather long molecular evolutionary lines can be detected by taking into consideration the three-dimensional structures, organic chemical reaction mechanisms and the appropriate chemotaxonomical data. The necessary chemical and biological data were taken from the Dictionary of Natural Products ${ }^{7}$ (in the following: DNP) occasionally completed by the data of the Chemical Abstracts on line and the Beilstein CrossFire databases. In the structural formulae and throughout the text, the biogenetic numbering system shown in structure of strictosidine 3 was consequently used. ${ }^{8}$ The only exceptions are the building blocks $\mathbf{1}$ and $\mathbf{2}$ which are numbered as indicated in their own formulae. 


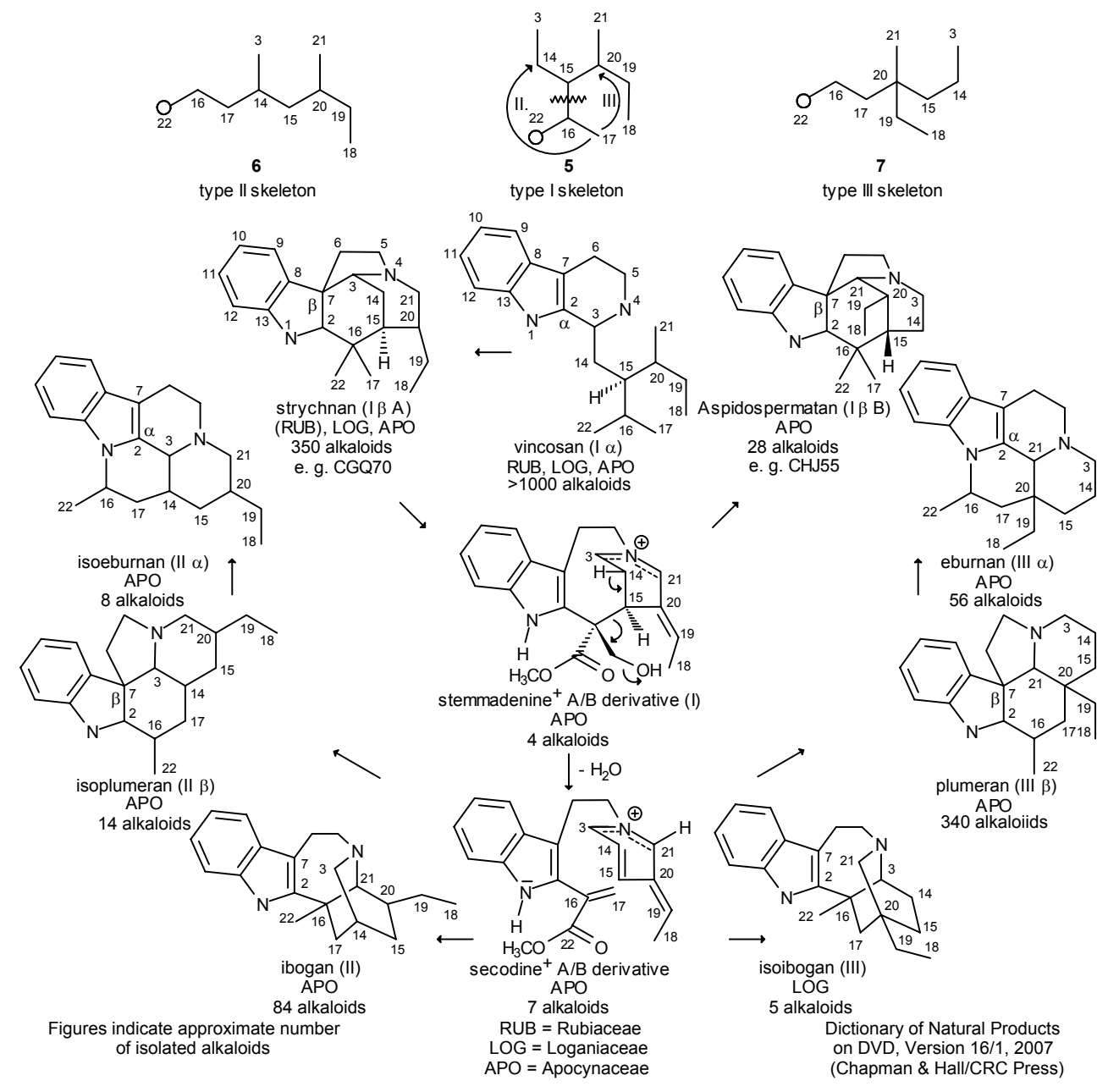

Scheme 2. Biogenetic-type system of indole alkaloids derived from secologanin.

\section{The biogenetic-type system of indole alkaloids}

In our previous study, 9,10 a biogenetic-type system of indole alkaloids (Scheme 2) was built up on the base of the three fundamental skeletons out of which secologanin is represented in these alkaloids. (In the schemes the number of the alkaloids in case can be given only approximatively, as it is changing by continuous renewal of the database.) Type I skeleton $\mathbf{5}$ contains the carbon frame in its original, type II 6 and type III 7 in a rearranged form. ${ }^{11}$ The generation starts with strictosidine corresponding to vincosan 8 i. e. to the basic skeleton of the type I $\alpha$ indole alkaloids in which C-3 (analogous to C-7 in secologanin) is attached to $\alpha$ position (C-2) of the indole ring. After deglucosylation, the hypothetic aglucone (represented as all-oxo aglucone 4 in scheme 1) undergoes several types of cyclizations and subsequent transformations. The main types of cyclization (scheme 3 ) take place between the nucleophilic $\mathrm{N}-4$ centre and one of the four electrophilic centres C-17, C-19, C-21 and C-22 of the secologanin subunit, and affords the vallesiachotaman 9, malindan 10, corynanthean 11 and vincoside lactam 12 skeletons, respectively. ${ }^{12}$ An additional cyclization between $\mathrm{C}-16$ and C-2 is 
directed, after subsequent 1,2-rearrangement of C-3 to C-7 ( $\beta$ position of the indole ring) to the formation of strychnan skeleton $\mathbf{1 3}$, which is the main type of the I $\beta$ A alkaloids. Cleavage of the C-3-C-7 bond in $\mathbf{1 3}$ gives the hypothetic monoseco stemmadenine ${ }^{+}$intermediate in which the way is opened partially by subsequent izomerization and recyclization toward the aspidospermatan alkaloids (I $\beta$ B), partially by further fragmentation to the bisseco secodine ${ }^{+}$ intermediate. This likewise hypothetic structure affords by further isomerization and two types of cyclizations the fused (isoplumeran II $\beta$ and plumeran III $\beta$ ) and the bridged (ibogan II and isoibogan III) alkaloids. Finally, the isoeburnan II $\alpha$ and eburnan III $\alpha$ skeletons are formed from the appropriate $\beta$ alkaloids by 1,2-rearrangement of C-3 or C-21, respectively, to centre C-2 (i. e. $\alpha$ position of the indole ring).

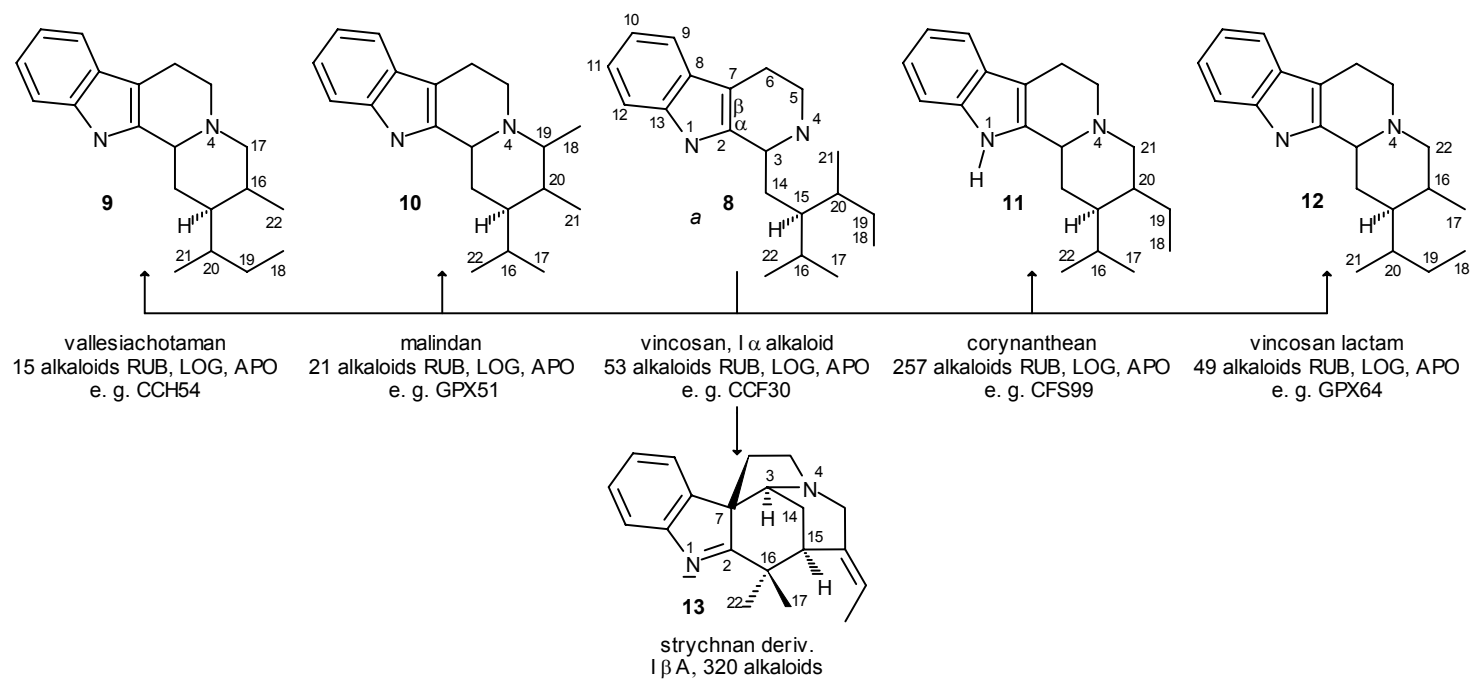

Scheme 3. Primary cyclizations in type I $\alpha$ indole alkaloids.

Generation of line strictosidine $1 \rightarrow$ stemmadenine $22 \mathrm{E} / \mathrm{O} \rightarrow$ ellipticine/olivacine 37/47 The study of this molecular evolutionary line was stimulated by the fact that the target alkaloids ellipticine 37 (in scheme 6) and olivacine 47 (in scheme 6) belong to a special group of indole alkaloids in which the type I skeleton 5 is intact, but the two carbon atoms C-5 and C-6 of the side-chain of the tryptamine subunit are removed, connection of the two subunits is strongly modified, and the two parent alkaloids are structural isomers, i. e. a methyl group is attached in $\mathbf{3 7}$ to the benzene and in $\mathbf{4 7}$ to the pyridine ring. The biogenetic numbering clearly shows these differences. ${ }^{13}$ Namely, the relation of the secologanin subunit and the N-4 atom corresponds in 37 to the corynanthean skeleton (11), in $\mathbf{4 7}$ to the malindan skeleton (10) (scheme 3). 


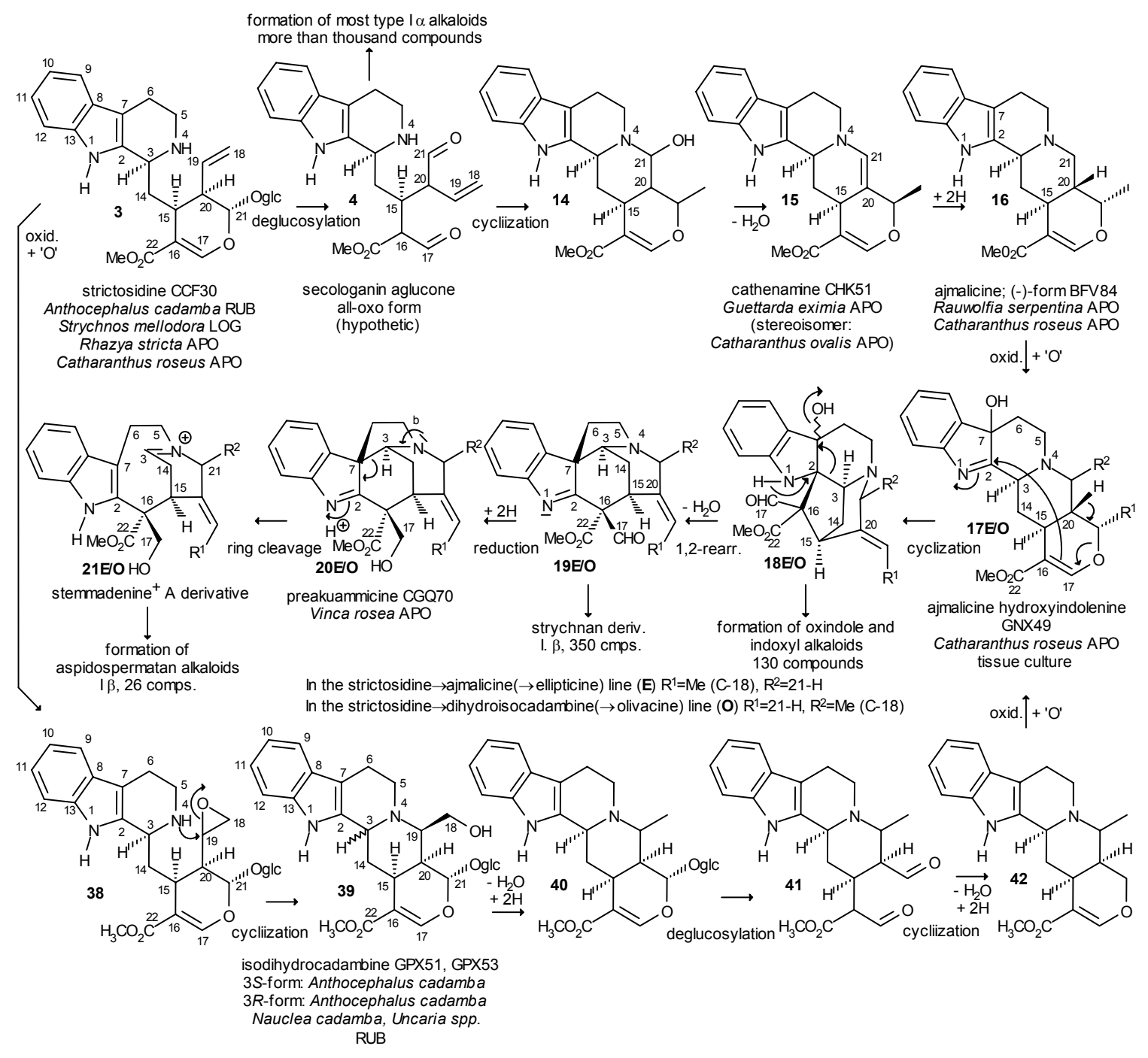

Scheme 4. Generation of ellipticine and olivacine. Part 1.

Derivation of ellipticine $\mathbf{3 7}$ is more straightforward. Enzymic deglucosylation of strictosidine 3 results in a multicomponent mixture of aglucones, which are represented also in Scheme 4 by the all-oxo form $4 .{ }^{14}$ One of these aglucones may be $\mathbf{1 4}$ which after elimination of water gives the alkaloid cathenamine 15 , and further by hydrogenation ajmalicine 16. Both of them belong to the corynanthean skeleton 11, and were isolated from natural sources. These types of alkaloids can be oxidized to different further alkaloids depending on the oxidation method. ${ }^{15}$ One of these products may be the ajmalicine hydroxyindolenine $17 \mathrm{E}$ isolated from Catharanthus roseus tissue culture. (In this and the following formulae of the line going to ellipticen alkaloids $(\mathbf{E}), \mathrm{R}^{1}=\mathrm{Me}$ $(\mathrm{C}-18), \mathrm{R}^{2}=21-\mathrm{H}$.) The DNP database contains several other hydroxyindolenine derivatives. The next isolated compound in this line is preakuammicine $20 \mathrm{E}$ obtained from Vinca rosea (=Cantharanthus roseus). Its formation from $17 \mathbf{E}$ can be interpreted by cyclization of C-16 to C2 in 18E, followed by a 1,2-rearrangement of C-3 to C-7 in 19E with elimination of water, and 
finally by reduction of the formyl group. $18 \mathrm{E}$ and $19 \mathrm{E}$ are starting points of a large number of oxindole and strychnan alkaloids, respectively.

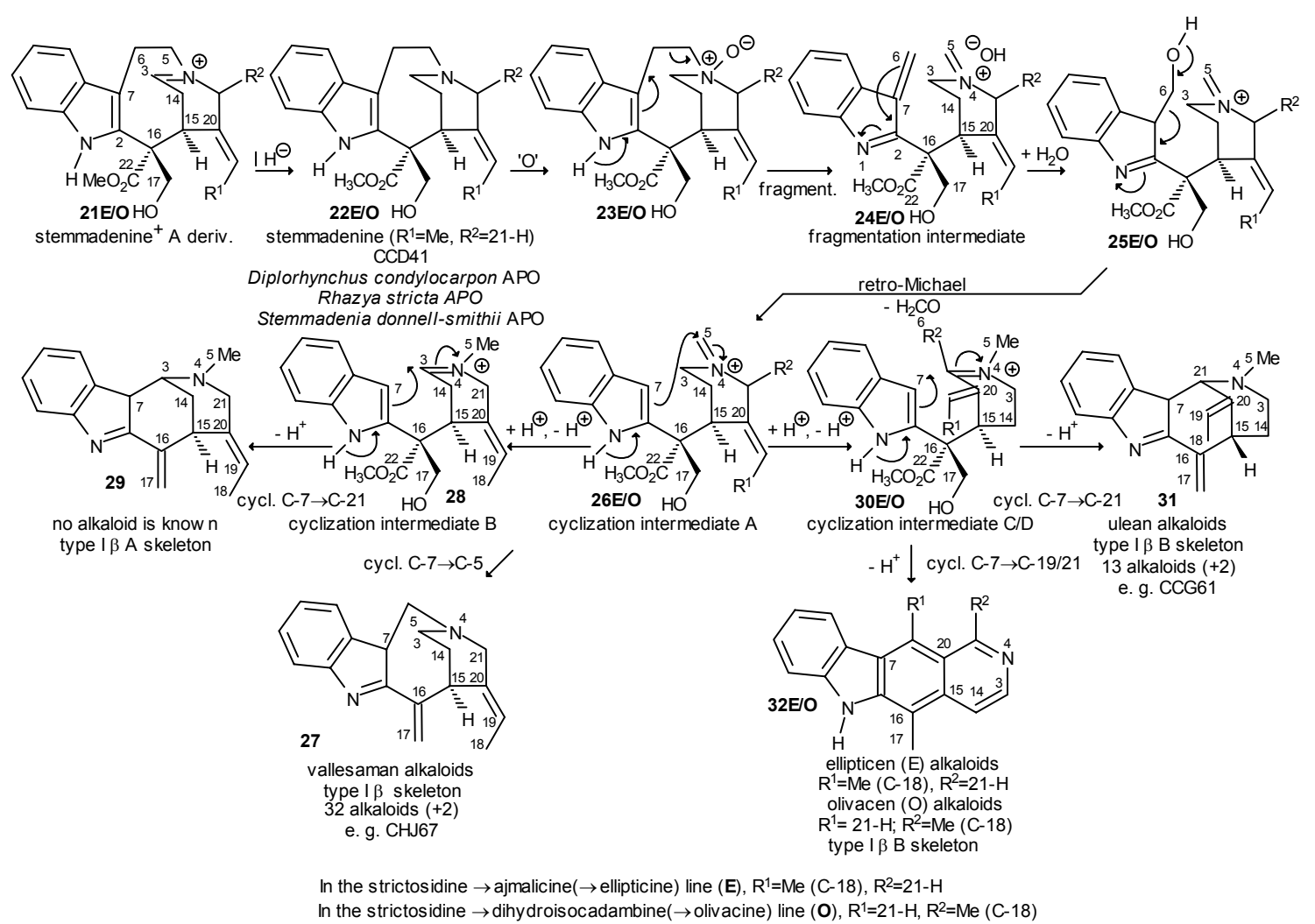

Scheme 5. Generation of ellipticine and olivacine. Part 2.

Preakuammicine $20 \mathrm{E}$ is an important point in the further evolution. The compound contains long-range interaction between the electron-releasing N-4 and the electron-attracting N-1 atoms (indicated by curved arrows in $\mathbf{2 0 E}$ ). Its further polarization by proton results in cleavage of the bond C-3-C-7. The positively charged and presumably highly reactive stemmadenine ${ }^{+}$A $\mathbf{2 1 E}$ structure was not isolated, but its reduced form stemmadenine 22E (in scheme 5) was obtained from natural sources. Stemmadenine ${ }^{+} \mathrm{A}$ can be izomerized by proton into stemmadenine ${ }^{+} \mathrm{B}$, in which recyclization in direction $\mathrm{C}-21 \rightarrow \mathrm{C}-7$ rather than $\mathrm{C}-3 \rightarrow \mathrm{C}-7$ affords the aspidospermatan (I $\beta$ B) alkaloids (shown in scheme 2). Stemmadenine 22E was isolated, among other species, from Rhazya stricta together with strictosidine and certain secodine derivatives (see later). This fact shows that the key compounds toward the evolution of ellipticine (and olivacine) were found in the same or related plant species. 


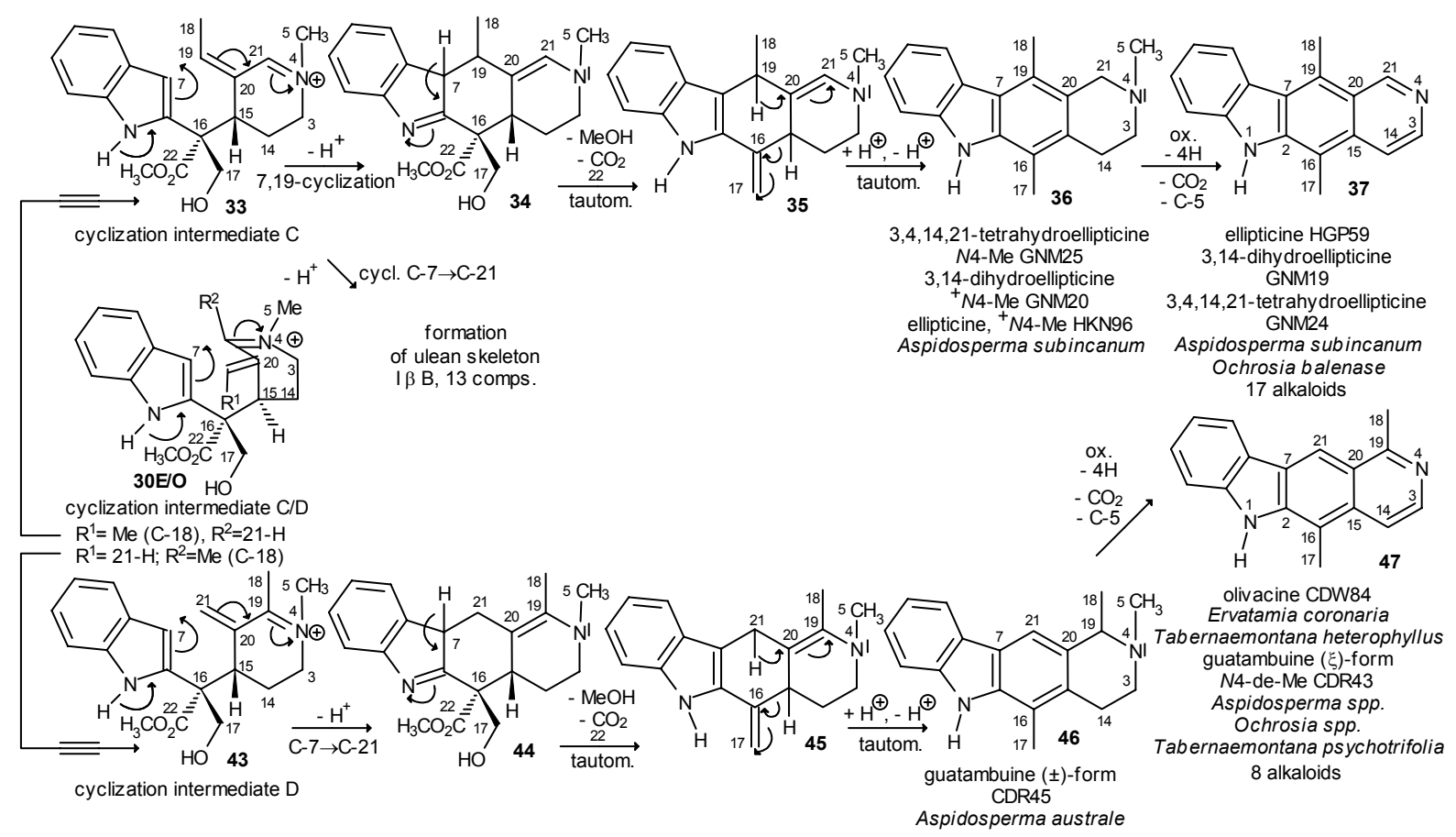

Scheme 6. Generation of ellipticine and olivacine. Part 3.

Stemmadenine $22 \mathrm{E}$ is the starting point of the changes in the side-chain of the tryptamine subunit of the type I $\beta$ indole alkaloids. These transformations are shown in Scheme 5. Stemmadenine 22E has the N-1 as a weakly electron-releasing centre, and its counterpart N-4 can be umpoled by formation of its N-4 oxide derivative 23E. The DNP database contains nearly hundred N-4 oxides dispersed in most subgroups. In $23 \mathbf{E}$ conditions are given for a reversed fragmentation (shown by curved arrows) to the fragmentation intermediate 24E. In all alkaloids having a truncated side-chain in the tryptamine subunit (nearly 50 natural products in the vallesaman 27, ulean 31, ellipticen 32E and olivacen $\mathbf{3 2 O}$ groups) C-6 is eliminated. The process can be interpreted by a retro Mannich reaction of the hydrated derivative $25 \mathrm{E}$ formed from $24 \mathrm{E}$. In the cyclization intermediate $\mathrm{A} \mathbf{2 6 \mathbf { E }}$ generated by that way, the $\mathrm{C}=\mathrm{N}$ double bond is developped between N-4 and C-5, and its immediate cyclization in direction C-7 $\rightarrow \mathrm{C}-5$ affords the vallesaman alkaloids 27. However, the C-3-N-4 double bond can be isomerized into the two neighbouring carbon atoms of the $\mathrm{N}-4$ atom of pyridine ring (C-3 and C-21/18 position, in cyclization intermediate $\mathrm{B} \mathbf{2 8 E}$ and $\mathrm{C} / \mathrm{D}$ 30E/O, respectively) as well. The conjugated position in cyclization intermediate $\mathrm{C} / \mathrm{D}$ is favoured over that of cyclization intermediate $\mathrm{B}$. Consequently, no natural product is known according to cyclization C-7 $\rightarrow$ C-3 in 29. From cyclization intermediate $\mathrm{C} / \mathrm{D} \mathbf{3 0 E} / \mathbf{O}$ further alkaloids can be generated, as it has two electrophilic centres at $\mathrm{C}-21$ and $\mathrm{C}-19$. The former is realized in the ulean 31, the latter in the ellipticen 32E alkaloids. As these processes were analyzed previously, ${ }^{10}$ at this place only the formation of the ellipticen alkaloids is briefly shown in the upper part of Scheme 6 . 
In the line going to the ellipticen alkaloids, deprotonation of the cyclization intermediate $\mathrm{C}$ 30E $\left(=33, \mathrm{R}^{1}=\mathrm{Me}(\mathrm{C}-18), \mathrm{R}^{2}=21-\mathrm{H}\right)$ gives already the tetracyclic structure 34 . The final steps toward ellipticen alkaloids $\mathbf{3 7}$ involve three types of tautomerization, elimination of methanol and carbondioxide (de(methoxycarbonylation), actually probably ester hydrolysis and decarboxylation) and dehydrogenation through 35 and 36. The driving force of the cascade of these reactions is evidently their increasing $\pi$-delocalization. The stepwise character of dehydrogenation could be demonstrated by isolation of the partially hydrogenated derivatives in 36. The closing oxidative demethylation $(36 \rightarrow 37)$ with elimination of C-5 propably runs in the tertiary immonium salt, which is supported by isolation of $N$-methyl-3,14-dihydroellipticine and $N$-methylellipticine, according to $\mathbf{3 6}$ and $\mathbf{3 7}$, respectively.

An additional remark should be added. Among the isolated ellipticen alkaloids there are two compounds in which the carbon atom of the ligands is on a higher oxidation state by one, i. e. 18-hydroxyellipticene and 17-oxoellipticene. Their isolation from natural sources suggests that in both cases one reductive step falls out in their formation. In case of 17-oxoellipticine, this step is the reduction of the formyl group at C-16 (step $19 \mathbf{E} \rightarrow \mathbf{2 0 E}$ ), and in case of 18hydroxyellipticene, the 20,21-double bond was not saturated (step $\mathbf{1 5} \rightarrow \mathbf{1 6}$ ). In this latter case, in the subsequent structures from 18E on, a 18,19,20,21-conjugated double bond system is present, which can be partially hydrated in a later state (probably before the final cyclization, $i$. e. step $33 \rightarrow \mathbf{3 4}$ ) of the molecular evolution.

As it was mentioned previously, 8 alkaloids of the isomeric olivacen skeleton (32O in scheme 5) were also isolated from natural sources. When the molecular evolution of ellipticine and olivacine is paralelly followed stepwise back to strictosidine $\mathbf{3}$, it might be concluded that the extra methyl group in these alkaloids should have been transposed formally from C-19 to C20 , i. e. it should be $\mathrm{C}-21$. Of course, such transposition cannot be rationalized in the frame of the present biogenetic principles. However, such a skeleton might be formed, when at the very beginning of the molecular evolution, the primary cyclization of $\mathrm{N}-4$ takes place at $\mathrm{C}-19$ rather than C-21 (see scheme 3). The skeleton formed by that way corresponds to the malindan (10) rather, than the corynanthean (11) structure. Really, this skeleton exists in isodihydrocadambine (39 in scheme 4) and several other alkaloids (altogether nearly 40 natural products developed from it). 39 is a glucoside, like strictosidine, and unlike ajmalicine, which means that the malindan skeleton can be formed before deglucosylation. The fact that $\mathbf{3 9}$ was isolated from Anthocephalus cadamba of the Rubiaceae family together with strictosidine 3, and its C-18 has a hydroxy group, suggests that it could be generated from strictosidine by epoxidation of the 18,19-double bond (38) followed by a nucleofilic attack of N-4 to 39 (lower part of scheme 4). These circumstances together with the additional fact that both ellipticen and olivacen alkaloids were isolated from the same genera Aspidosperma and Ochrosia (though not from the same species) (Apocynaceae) allows to take up mainly analogous common steps in their formation. These are shown in schemes 4 (steps $\mathbf{1} \rightarrow \mathbf{3 8 - 4 2} \rightarrow \mathbf{1 7 0 - 2 1 0}$ ), in scheme 5 (steps 210-260 $\rightarrow$ 30O $\rightarrow$ 32O) and in scheme 6 (steps $300 \rightarrow$ 43-47). (In the formulae of the line going to the olivacen alkaloids $(\mathbf{O}), \mathrm{R}^{1}=21-\mathrm{H}, \mathrm{R}^{2}=\mathrm{Me}(\mathrm{C}-18)$.) However, in the line going to olivacine, 
alkaloid groups analogous to the strychnan, aspidosospermatan, stemmadenine and secodine alkaloids as well as the type II and III alkaloids were not found as natural products.

\section{Generation of line strictosidine $1 \rightarrow$ vincadifformine $58 \rightarrow$ rhazinilam/rhazinal 64/73}

In this line a different challenge was presented to our studies. In the large and diversified group of the plumeran type (III $\beta$ ) alkaloids (more than 350 natural products) (Scheme 2) there is a small set of compounds, in which the bond C-2-C-7 of the tryptamine subunit is cleaved. The nine representatives of it (rhazinilam 64 ( in scheme 10), leuconolam and related compounds) have a quaternary carbon atom in their secologanin subunit, which suggested the presence of type III skeleton 7. Neither of these alkaloids has a methoxycarbonyl group $(=\mathrm{C}-22)$ attached to $\mathrm{C}-16$, however, one of them, rhazinal 73 has a formyl group at C-5. It may be supposed that its carbon atom corresponds to $\mathrm{C}-22$, however, its unusual position presented some difficulties for the interpretation of its generation. The first task was to derive the parent structure of these alkaloids (according to 64), followed by a second one to interpret the origin and unusual position of the formyl group in rhazinal 73.

The general molecular evolution going to the plumeran alkaloids is given in scheme 7 . The starting point is the charged stemmadenin ${ }^{+}$A 21E. If its 17-hydroxy group were transformed into a good living group (e. g. by acetylation, protonation or attachment to an enzyme), a fragmentation could start (according to the curved arrows in $\mathbf{2 1 E}$ ), secodine ${ }^{+} \mathrm{A} \mathbf{4 8}$ be formed and subsequently isomerized into secodine ${ }^{+}$B 49 in a deprotonation-reprotonation process. The driving force of this fragmentation is extension of the conjugation and the probable positive (or at least slightly negative) entropy. In secodine B 50B generated by deprotonation of 49, the conjugated double bonds can undergo a Diels-Alder reaction indicated by curved arrows, and immediately 18,19-didehydrotabersonine 51, the first member of the plumeran series should be formed.

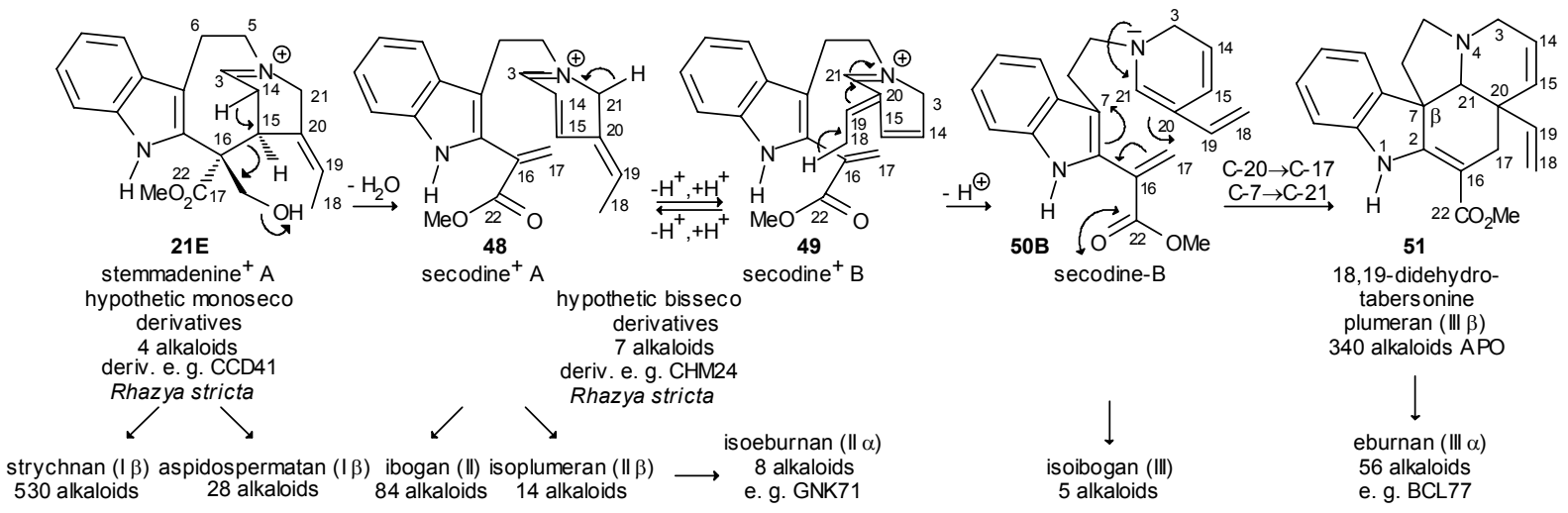

Scheme 7. Generation of rhazinalam and rhazinal. Part 1.

The whole matrix of transformations was analyzed previously ${ }^{6}$, and summarized briefly in 
Scheme 8. Unfortunately, 48 and 49, as well as 50A-D are hypothetic, probably highly reactive intermediates, and their existence could not be demonstrated directly. However, several partially hydrogenated secodine alkaloids were isolated, among other species, from Rhazya stricta. In 50A-D, types of conjugated double bond systems are found which can undergo Diels-Alder reactions through highly polarized transition states indicated by curved arrows, and afford the first representatives of the isoplumeran, plumeran, ibogan and isoibogan alkaloids, respectively. The actual mechanism of these transformations are likewise unknown. However, by the supposed way, a large number of isolated alkaloids (probably more than 500) could be derived from the indicated structures (mainly from 51, and except the isoibogan structure having only 5 alkaloids isolated till now), several of which contain unsaturation exactly at the sites and levels required by the supposed Diels-Alder reactions.

For the further molecular evolution toward rhazinilam 63, it is necessary to cleave bond C-7$\mathrm{C}$-21. In scheme 9 a further long-range interaction is shown by curved arrows in tabersonine and eburenin derivatives $\mathbf{5 2}$ and 53, respectively, which will probably be effective in the presence of proton. In this further key fragmentation the charged intermediates 54 and 55 should be formed, which are again hypothetic structures, however, their hydrogenated representatives in the vincadine 56 and quebrachamine 57 derivatives (14 and 20 alkaloids, respectively) are well known. Some quebrachamine derivatives were isolated even from Rhazya stricta.

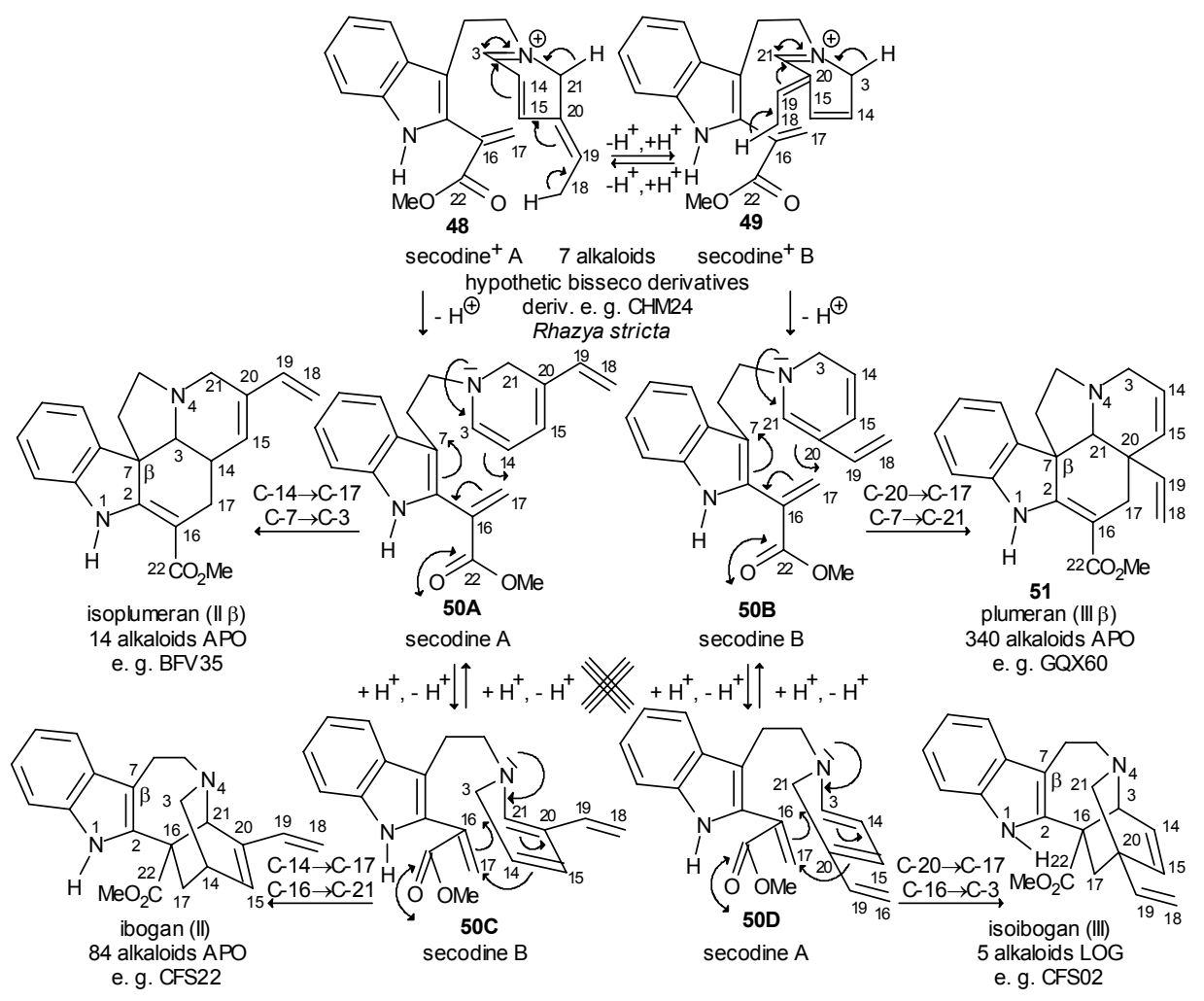

Scheme 8. Formation and transformation of the secodine structure. 


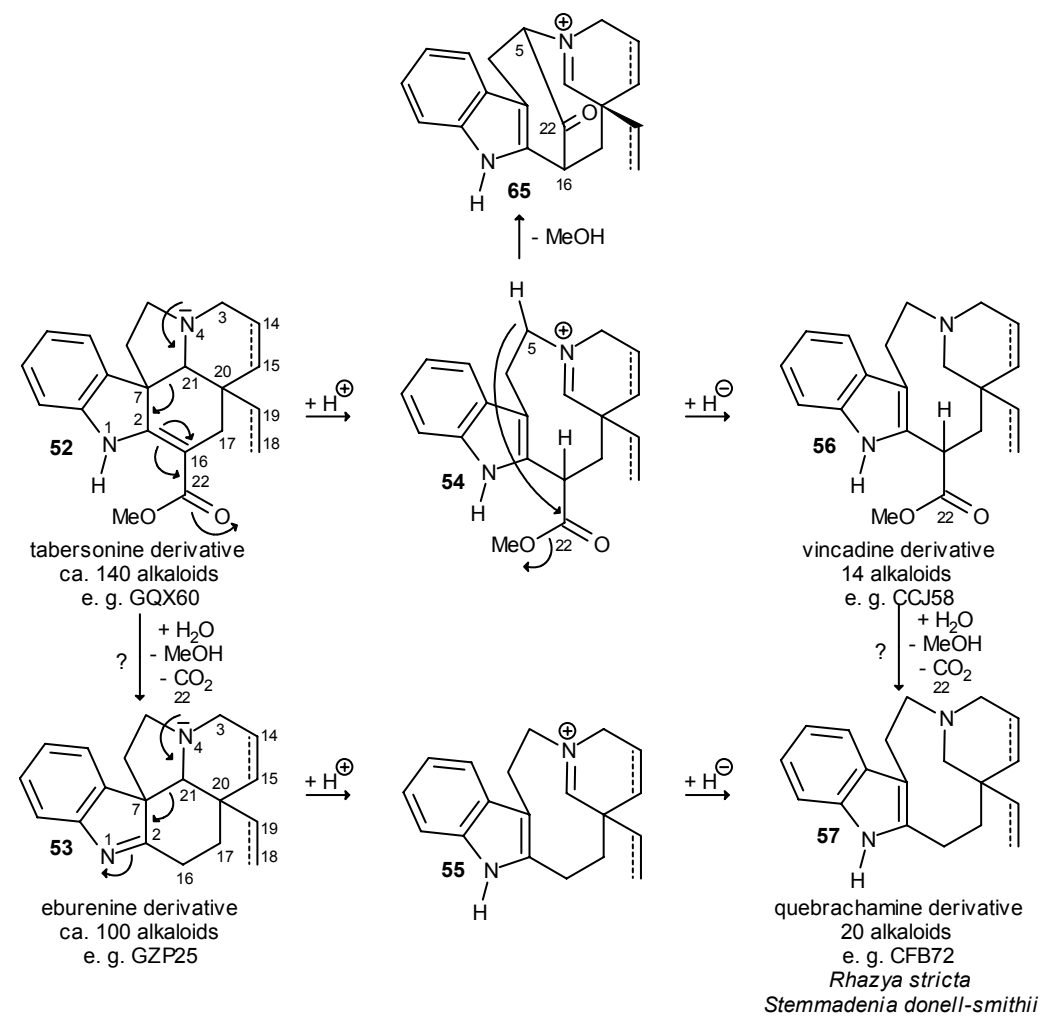

Scheme 9. Long-range interaction in the plumeran skeleton.

The fact that the methoxycarbonyl group is absent in rhazinilam 64 and related alkaloids suggests a de(methoxycarbonylation) somewhere in the molecular evolutionary line. Unfortunately it is not known if it takes place before or/and after the fragmentation. In the demethoxycarbonylated derivatives, the original double bond at $\mathrm{C}-2-\mathrm{C}-16$ is automatically moved to N-1-C-2 position conjugated to the benzene ring. In addition, the double bonds at position 18,19 and 14,15 of $\mathbf{5 1}$ and their derivatives, can partially be removed later by hydrogenation or other usual transformations of the olefinic elements. In the charged intermediates $\mathbf{5 4}$ and $\mathbf{5 5}$, the positive charge can temporarily be neutralized by a nucleofile from the proper solvent. It should be added that in the presence of ligand C-22, a further alternative is opened for the molecular evolution from 54 to 65 (see later).

Formation of rhazinilam 64 is shown in the lower part of scheme 10. The starting point would be vincadifformine 58, which can be formed by hydrogenation from the first plumeran compound 51. After de(methoxycarbonylation), in eburenin 59 the bond C-7-C-21 should be cleaved with formation of the charged intermediate 60. The C-2-C-7 double bond of it could be oxidized into a vicinal dihydroxy group in 61. This type of reaction is known in the indole alkaloids having an intact pyrrol ring, and several analogous alkaloids are found in the DNP database. By removal of a proton accompanied by a 1,2-rearrangement, C-7 can migrate from C2 to $\mathrm{C}-21$ with simultaneous formation of a nine-membered ring in $\mathbf{6 2}$ characteristic for the 
derivatives of rhazinilam. Dehydration in $\mathbf{6 2}$ with formation of a double bond in conjugation to the benzene ring provides already a natural product $\mathbf{6 3}$, and further dehydrogenation completes the formation of rhazinalam 64 itself. Further simple transformations not shown in the scheme afford the leuconolam derivatives.

However, there is a second, more challenging problem. It is highly improbable that in rhazinal 73 a functionalized carbon ligand (C-22) would disappear from position C-16, and a similar one appear at $\mathrm{C}-5$ from a source which would not be secologanin. A more satisfying solution would be the internal transposition of C-22 from C-16 to C-5, i. e. by cleavage of bond C-16-C-22 und construction of a new one C-5-C-22. The first suggestion to such a solution came from the observation that unlike other derivatives of rhazinilam 64, rhazinal 73 was isolated from a malayan Kopsia species. The typical alkaloids of the Kopsia genus are the kopsan alkaloids (more than 40 compounds), and their characteristic structural element is a onecarbon bridge (C-22) between C-6 and C-16. In scheme 9, an analogous possibility is opened in the charged tabersonine intermediate $\mathbf{5 4}$ in which $5-\mathrm{H}$ is activated by the immonium character of $\mathrm{N}-4$, and a one-carbon bridge (C-22) might be constructed between C-5 and C-16 in $\mathbf{6 5}$. This analogy suggested the derivation of rhazinal $\mathbf{7 3}$ along the line applied in the formation of rhazinilam 63, with intercalation of the bridge-making and bridge-braking steps necessary for transposition of C-22 from C-16 to C-5.

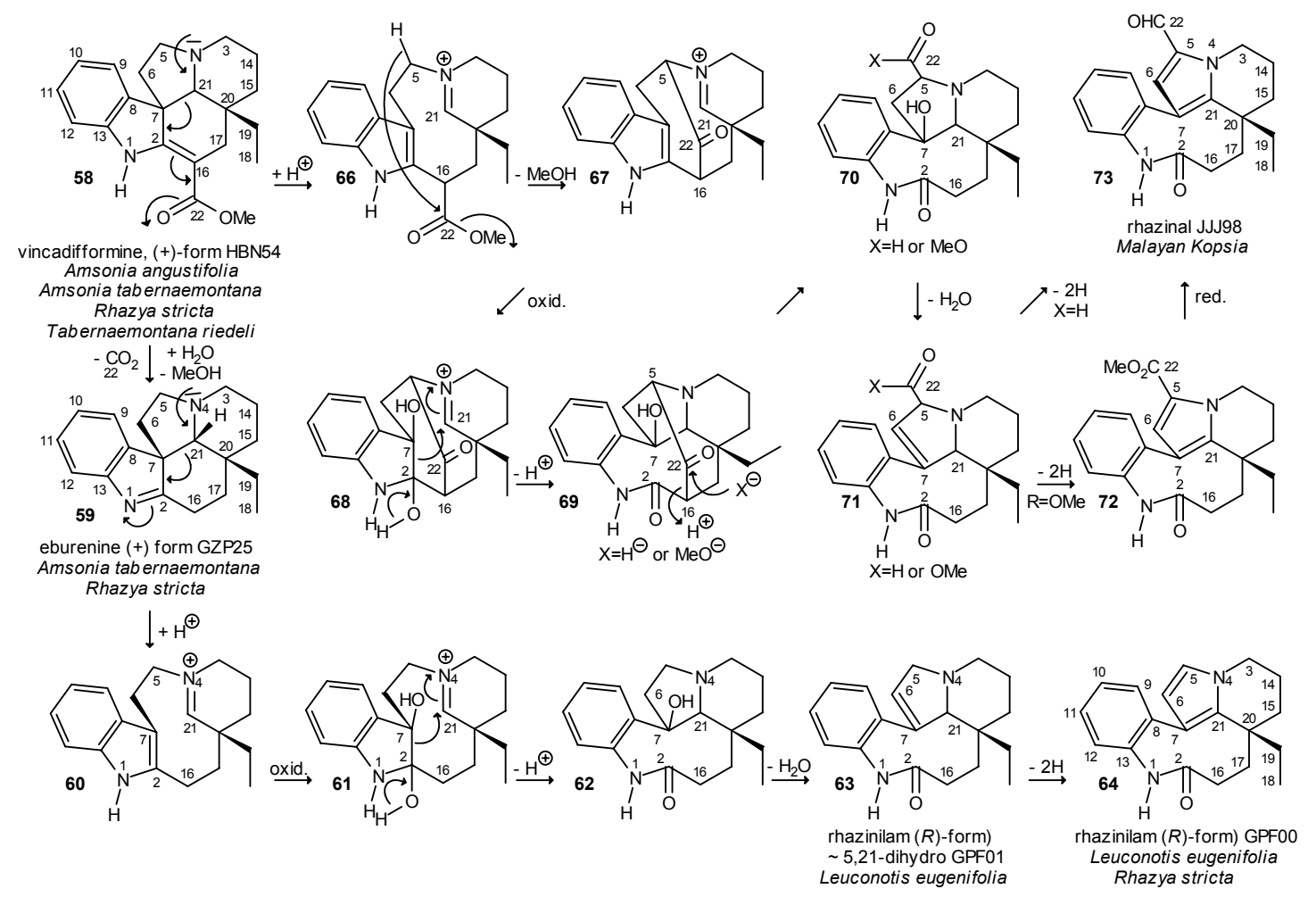

Scheme 10. Generation of rhazinilam and rhazinal. Part 2. 
According to the upper part of scheme 10, the development of rhazinal $\mathbf{7 3}$ would start likewise with vincadifformine 58. However, its fragmentation should run without de(methoxycarbonylation), and in $\mathbf{6 6}$ the structural conditions of the bridge formation are already given. Cyclization to $\mathbf{6 7}$ might formally be catalyzed by proton. The next two steps $\mathbf{6 7}$ $\rightarrow 68 \rightarrow 69$ are analogous to steps $60 \rightarrow 61 \rightarrow 62$ in the generation of rhazinilam. However, in 69 the amide carbonyl is a part of a $\beta$-dicarbonyl system and the bond C-16-C-22 could be cleaved by a nucleophile. Further steps $70 \rightarrow 71 \rightarrow 72$ (dehydration and dehydrogenation) are again analogous to steps $\mathbf{6 2} \rightarrow \mathbf{6 3} \rightarrow \mathbf{6 4}$. Were the nucleophile a hydride anion in step $\mathbf{6 9} \rightarrow \mathbf{7 0}$, C-22 would get immediately the formyl oxidation level. Were the nucleophile a methanolate ion, the C-22 would be part of a methoxycarbonyl group which should be reduced directly, or through the hydroxymethyl level and partial reoxidation to the formyl group in $\mathbf{7 3}$.

Unfortunately, rhazinal is a single compound without other known tightly related structures and was isolated only from an undefined "malayan" species. However, the chemistry in its formation as proposed above shows further analogies observed in the generation of other kopsan alkaloids or isolated from other Kopsia species. Such analogies are the bridge formation mentioned above (step $66 \rightarrow \mathbf{6 7})$ and the rearrangement of a 1,2-dihydroxy system $(\mathbf{6 1} \rightarrow \mathbf{6 2}$ and $68 \rightarrow 69$ ) in the derivation of the melodan alkaloids (isolated partly from a malayan Kopsia species), ${ }^{16}$ and the cleavage of the $\beta$-dicarbonyl system (step $69 \rightarrow 70$ ) in the formation of chanofruticosan subgroup of the kopsan alkaloids (isolated from other Kopsia species). ${ }^{17}$

\section{Conclusions}

The conclusions are summarized in Table 1. It should be remarked that the alkaloids shown in the previous schemes were generally isolated from several species, however, Table 1 gives only the most characteristic one or two sources.

1. The molecular evolutionary line from strictosidine $\mathbf{3}$ to rhazinilam $\mathbf{3 7}$ could be derived with high reliability, as both the starting educt 3 and the end product $\mathbf{3 7}$ were isolated from the same species Rhazya stricta, and many of the intermediates or their narrow derivatives were isolated likewise from Rhazya stricta and/or Catharanthus roseus (=Vinca rosea).

2. Rhazinal $\mathbf{7 3}$ as a single compound of its own type was isolated only from a malayan Kopsia species but from Rhazya stricta not yet. However, its formyl group at C-5 was successfully derived from the methoxycarbonyl group at 16 in a bridge-making-bridge-braking reaction sequence.

3. The molecular evolution of ellipticine from secologanin was abundantly supported by isolated natural products or their tightly related derivatives. This derivation was enhanced by the fact that ellipticen and olivacen alkaloids were isolated from common Ochrosia and Aspidosperma genera (though different species). 
Table 1. Summary of the molecular evolutionary lines studied in this paper and their chemotaxonomic properties

1. strictosidine $^{\mathrm{a}, \mathrm{b}} \rightarrow$ cathenamine $^{\mathrm{c}} \rightarrow$ ajmalicine $^{\mathrm{c}} \rightarrow$ ajmalicine hydroxyindolenine $^{\mathrm{b}}$

$\rightarrow$ preakuammicine $^{\mathrm{c}} \rightarrow$ secodine derivatives $^{\mathrm{a}} \rightarrow$ vincadifformine $^{\mathrm{a}} \rightarrow$ quebrachamine $^{\mathrm{a}}$

$\rightarrow$ rhazinilam $^{\mathrm{a}}$

2. strictosidine $^{\mathrm{a}, \mathrm{b}} \rightarrow$ cathenamine $^{\mathrm{c}} \rightarrow$ ajmalicine $^{\mathrm{c}} \rightarrow$ ajmalicine hydroxyindolenine $^{\mathrm{b}}$

$\rightarrow$ preakuammicine $^{\mathrm{c}} \rightarrow$ secodine derivatives $^{\mathrm{a}} \rightarrow$ vincadifformine $^{\mathrm{a}} \rightarrow \rightarrow \rightarrow$ rhazinal $^{\mathrm{d}}$

3. strictosidine $^{\mathrm{a}, \mathrm{b}} \rightarrow$ cathenamine $^{\mathrm{c}} \rightarrow$ ajmalicine $^{\mathrm{c}} \rightarrow$ ajmalicine hydroxyindolenine $^{\mathrm{b}} \rightarrow$

$\rightarrow$ preakuammicine $^{\mathrm{c}} \rightarrow$ stemmadenine $^{\mathrm{a}} \rightarrow \rightarrow \rightarrow$ ellipticine $^{\text {e.f }}$

4. strictosidine $^{\mathrm{g}} \rightarrow$ isodihydrocadambine $^{\mathrm{g}} \rightarrow{\text { analogous steps as in line } 3 \rightarrow \text { olivacine }^{\mathrm{e}}}^{\mathrm{e}}$

Plant species (among others) (all from the Apocynaceae family, except Anthocephalus cadamba from the Rubiaceae family).

${ }^{\mathrm{a}}$ Rhazya stricta. ${ }^{\mathrm{b}}$ Catharanthus roseus (=Vinca rosea). ${ }^{\mathrm{c}}$ Catharanthus species. ${ }^{\mathrm{d}}$ Malayan Kopsia species. ${ }^{\mathrm{e}}$ Ochrosia species and Aspidosperma species. ${ }^{\mathrm{f}}$ Kopsia species. ${ }^{\mathrm{g}}$ Anthocephalus cadamba

4. Though both ellipticine and olivacine are type I indole alkaloids, they belong to different primary cyclization patterns (common corynanthean and special malindan, respectively). However, their minute structural difference consists only in the mutual exchange of a methyl group and a hydrogen atom. Therefore, as a consequence of the tight structural similarities, the complete molecular evolutionary line could be derived with high reliability, though in the olivacine line only the identity of the final product and the chemotaxonomic relation of the starting precursor isodihydrocadamine with strictosidine could be established. This result could not have been obtained without the high potency of the computer-assisted searching of the large databases.

5. Several long-range bond-braking-bond-making interactions between the two nitrogen atoms $\mathrm{N}-1$ and N-4 have a key role in the construction of molecular evolutionary lines of indole alkaloids derived from secologanin.

6. Some plant species (e. g. Kopsia species) construct only a few very special types of alkaloids, however, Rhazya stricta, Catharanthus roseus and several other species provide natural products in nearly all classes of indole alkaloids.

\section{Acknowledgements}

The generous offering of the availability of the Dictionary of Natural Products database by the Chapman\&Hall/CRC is gratefully acknowledged. Without this help, the present study could not have been performed. The personal discussions with J. Buckingham strongly contributed to the solution of some special problems. 


\section{References}

1. Atta-Ur-Rahman, Anwer B. Biosynthesis of Indole Alkaloids, Clarendon Press: Oxford, 1983.

2. Cordell, G. A. Lloydia 1974, 37, 219.

3. Kisakürek, M. V.; Leeuwenberg, A. J.; Hesse, M. Alkaloids: Chemistry and Biological Properties, Vol. 1, Pelletier, S. W. Ed., John Wiley and Sons: New York, 1983, pp 211-376.

4. Hesse, M. Alkaloids, Verlag Helvetica Chimica Acta, Zürich, 2002.

5. Beke, Gy.; Patthy-Lukáts, Á.; Podányi, B; Szabó, L. F. Chirality 2001, 13, 483.

6. Beke, Gy.; Károlyházy, L.; Patthy-Lukáts, Á.; Podányi, B.; Szabó.L. F.: Chirality Transfer in the Formation of Indole Alkaloids Derived from Secologanin. In Progress in Biological Chirality, Palyi, G.; Zucchi, C.; Caglioti, L. Eds., Elsevier, Oxford (GB), 2004, pp 377.

7. Dictionary of Natural Products on DVD, Version 16.1, Chapman and Hall/CRC, Boca Raton, 2007. (In many cases $\mathrm{C} \& \mathrm{H}$ reference numbers are added to the names of the alkaloids, in order to facilitate the availability the abundant literature source as well as the CAS Registration Numbers in the compund entries of the DNP data base.)

8. Le Men, J.; Taylor, W. I. Experientia 1965, 21, 508.

9. Szabó, L. F. Studies in Natural Products Chemistry, Vol. 26. Bioactive Natural Products. Part G. Atta-Ur-Rahman Ed. Elsevier Science B. V. 2002, pp 95-148.

10. Szabo, L. F. Diversity and Selectivity in Molecular Evolution. In: Fundamentals of Life Palyi, G.; Zucchi, C.; Caglioti, L. Eds., Elsevier Life Science: Paris, 2002, pp 437-449.

11. Szabó, L. F. J. Phys. Org. Chem. 2006, 19, 579.

12. Károlyházy, L., Patthy-Lukáts, Á., Szabó, L. F. J. Phys. Org. Chem. 1998, 11, 622.

13. Differently from the DNP database, the biogenetic numbering system was retained even in the ellipticen and olivacen alkaloids. The parent names ellipticen and olivacen (without suffix 'e') concern the C-methylated tetracyclic ringsystem having, according to the IUPAC rules, the maximum number of non-cumulated double bonds (32/E and 32/O, respectively, in scheme 5). The individual alkaloids 37 and $\mathbf{4 7}$ have the names ellipticine and olivacine, respectively, according to the general practice.

14. Schwartz, A., Szabó, L. F., Podányi, B. Tetrahedron 1997, 53, 10489.

15. Ref. 1, p. 72-74.

16. Szabó L. ARKIVOC 2007, (vii), 280.

17. Results to be published. 